\title{
ONE-DIMENSIONAL GALERKIN METHODS AND SUPERCONVERGENCE AT INTERIOR NODAL POINTS*
}

\author{
MIENTE BAKKER $\dagger$
}

\begin{abstract}
In the case of one-dimensional Galerkin methods the phenomenon of superconvergence at the knots has been known for years [5], [7]. In this paper, a minor kind of superconvergence at specific points inside the segments of the partition is discussed for two classes of Galerkin methods: the Ritz-Galerkin method for $2 m$ th order self-adjoint boundary problems and the collocation method for arbitrary $m$ th order boundary problems. These interior points are the zeros of the Jacobi polynomial $P_{n}^{m, m}(\sigma)$ shifted to the segments of the partition; $n=k+1-2 m$, where $k$ is the degree of the finite element space. The order of convergence at these points is $k+2$, one order better than the optimal order of convergence. Also, it can be proved that the derivative of the finite element solution is superconvergent of $O\left(h^{k+1}\right)$ at the zeros of the Jacobi polynomial $P_{n+1}^{m-1, m-1}(\sigma)$ shifted to the segments of the partition. This is one order better than the optimal order of convergence for the derivative.
\end{abstract}

Key words. Galerkin methods, collocation methods, finite element method, superconvergence, Jacobi polynomials

AMS MOS subject classification. $65 \mathrm{~N} 30$

CR category. 5.17

1. Introduction. We consider the two-point boundary problem

$$
-\left(p(x) y^{\prime}\right)^{\prime}+q(x) y=f(x), \quad x \in[-1,+1]=I, \quad y( \pm 1)=0,
$$

where $p(x)>0, q(x) \geqq 0$ and $f(x)$ are sufficiently smooth. Let

$$
\begin{aligned}
& \Delta=\left\{-1=x_{0}<x_{1}<\cdots<x_{N}=1\right\}, \\
& x_{j}=-1+h j, \quad j=0, \cdots, N, \quad h=2 / N, \\
& I_{j}=\left[x_{j-1}, x_{j}\right], \quad j=1, \cdots, N
\end{aligned}
$$

be a uniform partition of $I$ and define $M_{0}^{k, 0}(\Delta)$ by

$$
M_{0}^{k, 0}(\Delta)=\left\{V \mid V \in C^{0}(I) ; V \in P_{k}\left(I_{j}\right), j=1, \cdots, N ; V( \pm 1)=0\right\}
$$

where for any interval $E, P_{k}(E)$ denotes the space of polynomials of degree $k$ restricted to $E$. Then the finite element approximation $Y \in M_{0}^{k, 0}(\Delta)$ of $y$ is determined by

$$
\left(p Y^{\prime}, V^{\prime}\right)+(q Y, V)=(f, V), \quad V \in M_{0}^{k, 0}(\Delta),
$$

where $(\cdot, \cdot)$ denotes the $L^{2}(I)$ inner product. It has the following convergence properties [7]

$$
\begin{array}{ll}
\|y-Y\|_{l} \leqq C_{1} h^{k+1-l}\|y\|_{k+1}, & l=0,1, \\
\left|(y-Y)\left(x_{j}\right)\right| \leqq C_{2} h^{2 k}\|y\|_{k+1}, & j=1, \cdots, N-1,
\end{array}
$$

where $C_{1}$ and $C_{2}$ are positive constants and where

$$
\begin{aligned}
\|v\|_{l} & =\left[\sum_{j=0}^{l}\left(D^{j} v, D^{j} v\right)\right]^{1 / 2}, \quad l \geqq 0, \\
D^{j} v & =\frac{d^{j} v}{d x^{j}}, \quad j \geqq 0 .
\end{aligned}
$$

* Received by the editors February 26, 1982, and in revised form March 15, 1983.

† Mathematisch Centrum, Box 4079, 1009 AB Amsterdam, the Netherlands.

101 
Also, it is known [3] that for specific points inside $I_{j}, Y$ has the error bound

$$
\begin{aligned}
& \left|(y-Y)\left(\xi_{j l}\right)\right| \leqq C(y) h^{k+2}, \\
& \xi_{j l}=x_{j-1}+\frac{h}{2}\left(1+\sigma_{l}\right), \quad l=1, \cdots, k-1, \quad j=1, \cdots, N,
\end{aligned}
$$

where $\sigma_{1}, \cdots, \sigma_{k-1}$ are the zeros of $P_{k}^{\prime}(\sigma)$ and $P_{k}(\sigma)$ is the $k$ th degree Legendre polynomial. This is one order better than the optimal error bound which is of $O\left(h^{k+1}\right)$.

It is this phenomenon of so-called interior superconvergence on which we will concentrate our attention. In the next two sections, we will treat two classes of finite element methods where this occurs: the Ritz-Galerkin and the collocation method [8]. Also, we will use that superconvergence to give a new proof of the superconvergence of the derivative at other Gaussian points [9].

Before that, we give some definitions we need throughout this paper.

For any $E \subset I$ and $m \geqq 0$, we define

$$
\begin{aligned}
\|v\|_{H^{m}(E)} & =\left[\sum_{l=0}^{m}\left(D^{l} v, D^{l} v\right)_{L^{2}(E)}\right]^{1 / 2}, \\
\|v\|_{W^{m}(E)} & =\sum_{l=0}^{m}\left\|D^{l} v\right\|_{L^{\infty}(E)}, \\
W^{m}(E) & =\left\{v \mid D^{l} v \in L^{\infty}(E), l=0, \cdots, m\right\} ; \\
H^{m}(E) & =\left\{v \mid D^{l} v \in L^{2}(E), l=0, \cdots, m\right\} .
\end{aligned}
$$

Also, we define the $\Delta$-related norms

$$
\begin{aligned}
& \|v\|_{m, \Delta}=\left[\sum_{j=1}^{N} \sum_{l=0}^{m}\left(D^{l} v, D^{l} v\right)_{L^{2}\left(I_{j}\right)}\right]^{1 / 2}, \\
& \|v\|_{W^{m}(\Delta)}=\max _{j=1, \cdots, N}\|v\|_{W^{m}\left(I_{j}\right)} .
\end{aligned}
$$

Finally, throughout this paper, $C, C_{1}$, etc. will be positive constants, not the same at each occurrence.

2. The Ritz-Galerkin method. Consider the $2 m$ th order two-point boundary problem

$$
\begin{aligned}
& L u \equiv \sum_{l=0}^{m}(-1)^{l} D^{l}\left[p_{l}(x) D^{l} u\right]=f(x), \quad x \in I, \\
& D^{l} u( \pm 1)=0, \quad l=0, \cdots, m-1,
\end{aligned}
$$

where $p_{0}, \cdots, p_{m}$ and $f$ are such that $u \in H^{s}(I)$, for some $s \geqq 2 m$, and that there exists some $C>0$ with the property

$$
\begin{aligned}
& B(v, v) \geqq C\|v\|_{m}^{2}, \quad v \in H_{0}^{m}(I), \\
& B(u, v)=\sum_{l=0}^{m}\left(p_{l} D^{l} u, D^{l} v\right), \quad u, v \in H_{0}^{m}(I), \\
& H_{0}^{m}(I)=\left\{v \mid v \in H^{m}(I) ; D^{l} v( \pm 1)=0, l=0, \cdots, m-1\right\}
\end{aligned}
$$

in other words, $B(\cdot, \cdot)$ is strongly coercive. 
For some partition $\Delta$ of $I$ defined by (1.2) and some integer $k \geqq 2 m-1$, we define the finite element space

$$
M_{0}^{k, m}(\Delta)=\left\{V \mid V \in H_{0}^{m}(I) ; V \in P_{k}\left(I_{j}\right), j=1, \cdots, N\right\} .
$$

The solution $u$ of (2.1) can be approximated in $M_{0}^{k, m}(\Delta)$ by the solution $U$ of the weak Galerkin form

$$
B(U, V)=(f, V), \quad V \in M_{0}^{k, m}(\Delta) .
$$

If $u \in H^{k+1}(I) \cap H_{0}^{m}(I)$, the error function $e=u-U$ has the bounds [2], [4]

$$
\begin{aligned}
& \|e\|_{l} \leqq C h^{k+1-l}\|u\|_{k+1}, \quad l=0, \cdots, m, \\
& \left|D^{l} e\left(x_{j}\right)\right| \leqq C h^{2 r}\|u\|_{k+1}, \quad l=0, \cdots, m-1, \quad j=1, \cdots, N-1, \\
& r=k+1-m .
\end{aligned}
$$

What we want to prove is the fact that inside each segment $I_{i}$ there exist $n=k+1-2 m$ distinct and specific points where $|e(x)|$ is of $O\left(h^{k+2}\right)$, one order better than the optimal order of convergence. This is, of course, only true, if $n \geqq 1$ or $k \geqq 2 m$. These points are shown to be the zeros of the Jacobi poynomial $P_{n}^{m, m}(\sigma)$, which will be introduced in the next section.

Remark. For reasons of convenience, we confine ourselves to the case that $L$ is a self-adjoint operator and that $\Delta$ is a uniform partition of $I$. The results in $\S 2$, however, are also valid if $\Delta$ is quasi-uniform (i.e. $\max h_{i} \geqq \lambda \min h_{i}, \lambda$ independent of the mesh) and if $L$ is a skew-adjoint operator of the form

$$
L u=\sum_{i=0}^{m}(-1)^{i} D^{i}\left[\sum_{i=0}^{i} p_{i j}(x) D^{i} u\right],
$$

provided that, of course $B($, ) defined by

$$
B(u, v)=\sum_{i=0}^{m} \sum_{j=0}^{i}\left(p_{i j} D^{j} u, D^{i} v\right)
$$

is strongly coercive.

2.1. The Jacobi polynomial. The Jacobi polynomial $P_{n}^{\alpha, \beta}(\sigma)$ is defined by Rodrigues' formula [1] as

$$
\begin{aligned}
& P_{n}^{\alpha, \beta}(\sigma)=[w(\sigma)]^{-1} D^{n}\left[\left(1-\sigma^{2}\right)^{n} w(\sigma)\right] A_{n}^{\alpha, \beta}, \quad n \geqq 0, \\
& w(\sigma)=(1-\sigma)^{\alpha}(1+\sigma)^{\beta}, \quad \alpha, \beta>-1,
\end{aligned}
$$

where $A_{n}^{\alpha, \beta}$ is some normalizing factor, e.g., such that $P_{n}^{\alpha, \beta}(1)=1$ or $P_{n}^{\alpha, \beta}(1)=$ $(1+\alpha)(1+\alpha / 2) \cdots(1+\alpha / n)$. It has the important property

$$
\left(w P_{i}^{\alpha, \beta}, P_{j}^{\alpha, \beta}\right)=\delta_{i j}\left(w P_{i}^{\alpha, \beta}, P_{i}^{\alpha, \beta}\right), \quad 0 \leqq i, j,
$$

where $\delta_{i j}$ is the Kronecker symbol.

From now on, we are only interested in the case $\alpha=\beta=m$, where $m$ is some nonnegative integer. In that case, we replace the double superscript $m, m$ by the single superscript $m$.

LEMMA 1. Let the linear interpolation $\Pi: C^{-1}(I) \rightarrow P_{n+2 m-1}(I)$ be determined by

$$
\begin{aligned}
& D^{l}(\Pi f)( \pm 1)=D^{l} f( \pm 1), \quad l=0, \cdots, m-1, \\
& (\Pi f)\left(\sigma_{i n}^{m}\right)=f\left(\sigma_{i n}^{m}\right), \quad i=1, \cdots, n,
\end{aligned}
$$


where $\sigma_{i n}^{m}, i=1, \cdots, n$ are the zeros of $P_{n}^{m}(\sigma)$. Then, for $f \in W^{2(m+n)}(I)$, we have the approximation

$$
\begin{aligned}
& \int_{-1}^{+1} f(\sigma) d \sigma=\int_{-1}^{+1}(\Pi f)(\sigma) d \sigma+R_{m n} D^{2 r} f(\xi), \xi \in(-1,+1), \\
& R_{m n}=(-1)^{m} 2^{2 r+1} \frac{n !(r !)^{2}(2 m+n) !}{[(2 r) !]^{3}(2 r+1)}, \\
& r=m+n=k+1-m .
\end{aligned}
$$

This is a generalization of Legendre $(m=0)$ and Lobatto quadrature $(m=1)$.

Proof. From (2.8), it follows that there exists a function $g(\sigma)$ such that

$$
(f-\Pi f)(\sigma)=\left(1-\sigma^{2}\right)^{m} P_{n}^{m}(\sigma) g(\sigma) .
$$

From the orthogonality relation (2.7), we learn that

$$
(f-\Pi f, I)=0, \quad \text { if } g \in P_{n-1}(I),
$$

which means that for $f \in P_{2 r-1}(I)$, the quadrature error is zero. For any other $f \in W^{2 r}(I)$, it is clear that

$$
\int_{-1}^{+1}(f-\Pi f)(\sigma) d \sigma=\int_{-1}^{+1}(f-F) d \sigma
$$

where $F \in P_{2 r-1}(I)$ is some Hermite approximation of $f$ which satisfies the relations (2.8) with $\Pi f$ replaced by $F$. The rest of the proof follows from the theory of Ciarlet and Raviart [6]. For the evaluation of $R_{m n}$ we refer to the appendix.

Elaboration of (2.8) gives the formula

$$
\int_{-1}^{+1}(\Pi f)(\sigma) d \sigma=\sum_{l=0}^{m-1}\left[\theta_{l 1} D^{l} f(-1)+\theta_{l 2} D^{l} f(+1)\right]+\sum_{l=1}^{n} \omega_{l} f\left(\sigma_{l n}^{m}\right)
$$

with

$$
\begin{aligned}
& \omega_{i}=\int_{-1}^{+1} \Phi_{i}(\sigma) d \sigma, \quad \Phi_{i}(\sigma)=\frac{\left(1-\sigma^{2}\right)^{m} P_{n}^{m}(\sigma)}{\left(\sigma-\sigma_{i n}^{m}\right)\left[\left(1-\sigma^{2}\right)^{m} d P_{n}^{m}(\sigma) / d \sigma\right]_{\sigma=\sigma_{i n}^{m}}}, \\
& \theta_{l i}=\int_{-1}^{+1} \psi_{l i}(\sigma) d \sigma, \quad \psi_{l i} \in P_{k}(I), \\
& \psi_{l i}\left(\sigma_{i n}^{m}\right)=0, \quad l=0, \cdots, m-1, \quad i=1,2, \quad j=1, \cdots, n, \\
& D^{s} \psi_{l i}\left((-1)^{j}\right)=\delta_{i j} \delta_{l s}, \quad 1 \leqq i, j \leqq 2, \quad \xi \leqq l, s \leqq m-1 .
\end{aligned}
$$

Note that in (2.13), $\Phi$ and $\Psi$ are natural basis functions for Hermite interpolation and $\sigma_{j n}^{m}, j=1, \cdots, n$ are the zeros of $P_{n}^{m}(\sigma)$.

In the next section, we will use (2.9)-(2.12) to establish superconvergence of $O\left(h^{k+2}\right)$ at the Jacobi points.

2.2. Superconvergence at Jacobi points. We return to problem (2.1) and its Ritz-Galerkin solution (2.4). It is standard that

$$
B(e, V)=0, \quad V \in M_{0}^{k, m}(\Delta) .
$$

For $k \geqq 2 m$, we define for any $I_{j}$ the $n$-dimensional subspace $S_{0}\left(I_{j}\right)$ of $M_{0}^{k, m}(\Delta)$ by

$$
S_{0}\left(I_{j}\right)=\left\{V \mid V \in H_{0}^{m}(I) \cap P_{k}\left(I_{j}\right) ; \operatorname{supp}(V)=I_{j}\right\} .
$$


For $S_{\mathrm{o}}\left(I_{j}\right)$, a basis can be constructed, consisting of the Lagrange polynomials $\phi_{i}(x)$ defined by

$$
\phi_{i}(x)=\Phi_{i}\left(1+2\left(x-x_{j}\right) / h\right), \quad i=1, \cdots, n,
$$

where $\Phi_{i}$ is defined by (2.13).

If we apply (2.14) to $\phi_{i}$, we obtain after partial integration

$$
\left(e, L \phi_{i}\right)=\sum_{l=1}^{m} \sum_{\nu=0}^{l-1}\left[(-1)^{\nu+1} D^{l-\nu 1} e(x) D^{\nu}\left(p_{l}(x) D^{l} \phi_{i}(x)\right)\right]_{x_{j-1}}^{x_{i}}, \quad i=1, \cdots, n .
$$

We now define the interior nodal points $\xi_{j l}$ by

$$
\xi_{j l}=x_{j-1}+\frac{h}{2}\left(1+\sigma_{l n}^{m}\right), \quad l=1, \cdots, n,
$$

where $\sigma_{l n}^{m}$ is the $l$ th zero of $P_{n}^{m}(\sigma)$, as defined in $\S 2.1$.

Application of Lemma 1 to $\left(e, L \phi_{i}\right)$ combined with the use of (2.17) gives

$$
\begin{aligned}
\frac{h}{2} \sum_{l=1}^{n} \omega_{l} e\left(\xi_{j l}\right) L \phi_{i}\left(\xi_{j l}\right)=\sum_{l=1}^{m} & \sum_{\nu=0}^{l-1}\left[(-1)^{\nu+1} D^{l-\nu-1} e(x) D^{\nu}\left(p_{l}(x) D^{l} \phi_{i}(x)\right)\right]_{x_{j-1}}^{x_{i}} \\
& -\sum_{l=0}^{m-1} \sum_{\nu=1}^{2} \theta_{l \nu} D^{l}\left(e L \phi_{i}\right)\left(x_{j-2+\nu}\right)\left(\frac{h}{2}\right)^{l+1} \\
& -\left(\frac{h}{2}\right)^{2 r+1} R_{m n}\left[D^{2 r}\left(e L \phi_{i}\right)\right]_{x=\xi \in I_{i}}, \quad i=1, \cdots, n,
\end{aligned}
$$

where $R_{m n}$ is defined by (2.9). If we multiply both sides of (2.19) by $2 h^{2 m-1}$ and apply formula (2.5), we have

$$
\begin{aligned}
\left|\sum_{l=1}^{n}\left[\omega_{l} L \phi_{i}\left(\xi_{j l}\right) h^{2 m}\right] e\left(\xi_{j l}\right)\right| & \leqq C_{1} \sum_{l=0}^{m-1}\left(\left|D^{l} e\left(x_{j-1}\right)\right|+\left|D^{l} e\left(x_{j}\right)\right|\right)+C_{2} h^{2 k+2}\left\|e L \phi_{i}\right\|_{W^{2 r}\left(I_{j}\right)} \\
& \leqq C_{1} h^{2 r}\|u\|_{k+1}+C_{2} h^{k+2}\|e\|_{W^{2 r}\left(I_{j}\right)} .
\end{aligned}
$$

We need an estimate of $\|e\|_{W^{2 r}\left(I_{j}\right)}$. To that end, we define the projection $\Pi_{\Delta}: H_{0}^{m}(I) \cap$ $W^{k+1}(I) \rightarrow M_{0}^{k, m}(\Delta)$ by

$$
\begin{aligned}
& \left(\Pi_{\Delta} u\right)\left(\xi_{j l}\right)=u\left(\xi_{j l}\right), \quad j=1, \cdots, N, \quad l=1, \cdots, n, \\
& D^{l}\left(\Pi_{\Delta} u\right)\left(x_{j}\right)=D^{l} u\left(x_{j}\right), \quad j=0, \cdots, N, \quad l=0, \cdots, m-1 .
\end{aligned}
$$

Then we have

$$
\begin{aligned}
& \|e\|_{W^{2 r}\left(I_{j}\right)} \leqq\|\varepsilon\|_{W^{2 r}\left(I_{j}\right)}+\|\delta\|_{W^{k}\left(I_{j}\right)}, \\
& \varepsilon=u-\Pi_{\Delta} u, \quad \delta=U-\Pi_{\Delta} u, \quad \delta \in M_{0}^{k, m}(\Delta) .
\end{aligned}
$$

Since for any $x \in I_{j}$, we have (see [6])

$$
\left|D^{l} \varepsilon(x)\right| \leqq \begin{cases}\left|D^{l}(u) x\right|, & l>k, \\ C h^{k+1-l}\left\|D^{k+1} u\right\|_{L^{\infty}\left(I_{j}\right)}, & l \leqq k,\end{cases}
$$

and since

$$
\begin{aligned}
\|\delta\|_{W^{k}\left(I_{j}\right)} & \leqq C h^{-k}\|\delta\|_{L^{\infty}\left(I_{j}\right)} \\
& \leqq C h^{-k}\left[\|e\|_{L^{\infty}\left(I_{j}\right)}+\|\varepsilon\|_{L^{\infty}\left(I_{j}\right)}\right] \quad \text { (Poincaré's inequality) } \\
& \leqq C h^{-k}\left[\|e\|_{1}+C_{1} h^{k+1}\|u\|_{W^{k+1}\left(I_{j}\right)}\right] \\
& \leqq C\left[\|u\|_{k+1}+C_{1} h\|u\|_{W^{k+1}\left(I_{j}\right)}\right],
\end{aligned}
$$


it turns out that

$$
\|e\|_{W^{2 r_{\left(I_{j}\right)}}} \leqq\|\varepsilon\|_{W^{2 r}\left(I_{j}\right)}+\|\delta\|_{W^{k}\left(I_{j}\right)} \leqq C\left[\|u\|_{k+1}+\|u\|_{W^{2 r}\left(I_{j}\right)}\right]
$$

and hence

$$
\left|\sum_{l=1}^{n}\left[\omega_{l} L \phi_{i}\left(\xi_{j e}\right) h^{2 m}\right] e\left(\xi_{j e}\right)\right| \leqq C h^{k+2}\left(\|u\|_{k+1}+\|u\|_{W^{2 r}\left(I_{j}\right)}\right) .
$$

Remark. In (2.24), we used the property

$$
h^{l}\|V\|_{W^{l}\left(I_{j}\right)} \leqq C h^{i}\|V\|_{W^{i}\left(I_{j}\right)}, \quad V \in P_{k}\left(I_{j}\right), \quad 0 \leqq i, l \leqq k .
$$

On the other hand, if we apply Lemma 1 to the inner product

$$
2 h^{2 m-1}\left(\phi_{l}, L \phi_{i}\right)=2 h^{2 m-1} B\left(\phi_{i}, \phi_{l}\right) \text {, }
$$

we find that

$$
\left|2 h^{2 m-1} B\left(\phi_{i}, \phi_{l}\right)-h^{2 m} \omega_{l} L \phi_{i}\left(\xi_{j l}\right)\right| \leqq C h^{2 k+2}\left\|\phi_{l} L \phi_{i}\right\|_{W^{2 r}\left(I_{j}\right)} \leqq C h^{2},
$$

which means that $\left(h^{2 m} \omega_{l} L \phi_{i}\left(\xi_{j l}\right)\right)$ is an $O\left(h^{2}\right)$ perturbation of a positive definite matrix whose entries are of $O(1)$.

If we present $\left(h^{2 m} \omega_{l} L \phi_{i}\left(\xi_{j e}\right)\right)$ and $\left(2 h^{2 m-1} B\left(\phi_{i}, \phi_{e}\right)\right)$ by $M_{1}$ and $M_{2}$ respectively, we find that, since $M_{2}^{-1}$ exists and is of $O(1)$ (this follows from the strong coercivity of $B$ ),

$$
M_{1}=M_{2}+h^{2} M_{3}=M_{2}\left(I+h^{2} M_{2}^{-1} M_{3}\right)=M_{2}\left(I+h^{2} M_{4}\right),
$$

where the entries of $M_{4}$ are of $O(1)$. Elementary matrix calculus shows that $(I+$ $\left.h^{2} M_{4}\right)^{-1}$ exists, if $h$ is small enough, and can be expanded in a power series:

$$
\left(I+h^{2} M_{4}\right)^{-1}=\sum_{l=0}^{\infty}(-1)^{l} h^{2 l} M_{4}^{l} .
$$

This implies that $\left(h^{2 m} \omega_{l} L \phi_{i}\left(\xi_{j l}\right)\right)^{-1}$ exists and has entries of $O(1)$. This completes the proof of

THEOREM 1. Let $u \in H_{0}^{m}(I) \cap H^{k+1}(I) \cap W^{2 r}(\Delta)$ be the solution of (2.1) and let $U \in M_{0}^{k, m}(\Delta)$ be the solution of (2.4). Then $e=u-U$ has the bounds (2.5) and the additional bound

$$
\left|e\left(\xi_{j l}\right)\right| \leqq C(u) h^{k+2}, \quad j=1, \cdots, N, \quad l=1, \cdots, n,
$$

where $\xi_{j l}$ is defined by (2.18).

We can use the local convergence properties (2.5) and (2.31) to establish superconvergence properties of $D e$ at interior points of $I_{j}$. Let $\varepsilon(x)$ be defined by (2.22). Then on any $I_{j}, \varepsilon(x)$ has the representation

$$
\begin{aligned}
& \varepsilon(x)=h^{k+1}\left(1-\sigma^{2}\right)^{m} P_{n}^{m}(\sigma) E_{j}(x), \\
& \sigma=\frac{2}{h}\left(x-\bar{x}_{j}\right), \quad \bar{x}_{j}=\frac{1}{2}\left(x_{j-1}+x_{j}\right),
\end{aligned}
$$

where $E_{j}(x)$ and $E_{j}^{\prime}(x)$ have bounds depending on $j$ only. This property can be proved by expanding $u$ and $\Pi_{\Delta} u$ as Taylor series around $\bar{x}_{j}$.

Differentiating (2.32), we obtain

$$
\varepsilon^{\prime}(x)=h^{k+1} E_{j}^{\prime}(x)\left(1-\sigma^{2}\right)^{m} P_{n}^{m}(\sigma)+2 h^{k} E_{j}(x) \frac{d}{d \sigma}\left(1-\sigma^{2}\right)^{m} P_{n}^{m}(\sigma) .
$$


From (2.6) and [1, Formula 22.6.1], it can be proved that

$$
\begin{aligned}
& P_{n}^{m}(\sigma)=A_{m n} \frac{d}{d \sigma} P_{n+1}^{m-1}(\sigma), \\
& \frac{d}{d \sigma}\left[\left(1-\sigma^{2}\right)^{m} \frac{d}{d \sigma} P_{m+1}^{m-1}(\sigma)\right]=B_{m n}\left(1-\sigma^{2}\right)^{m-1} P_{n+1}^{m-1}(\sigma),
\end{aligned}
$$

where $A_{m n}$ and $B_{m n}$ depend on $m$ and $n$ only. From (2.33) and (2.34) we can conclude that

$$
\begin{aligned}
& \left|\varepsilon^{\prime}(x)\right|=O\left(h^{k+1}\right) \quad \text { if } x=\eta_{j l}, \\
& \eta_{j l}=x_{j-1}+\frac{h}{2}\left(1+\sigma_{l n+1}^{m-1}\right), \quad j=1, \cdots, N, \quad l=1, \cdots, n+1 .
\end{aligned}
$$

Consider now $\delta$ defined by (2.22). From (2.5) and Theorem 1, it is proved that

$$
\|\delta\|_{L^{\infty}(I)} \leqq C(u) h^{k+2}, \quad\left\|\delta^{\prime}\right\|_{L^{\infty}(I)} \leqq C(u) h^{k+1} .
$$

From (2.35)-(2.36), one easily proves bound

THEOREM 2. Let the conditions of Theorem 1 hold. Then $e(x)$ has the additional

$$
\left|D e\left(\eta_{j l}\right)\right| \leqq C(u) h^{k+1},
$$

where $\eta_{j l}$ is defined by (2.35). This is one order better than the optimal order of convergence for $e^{\prime}(x)$.

2.3. Quadrature rules. Without giving proofs, we state that all the local convergence properties from the Theorems 1 and 2 are preserved whenever $(\cdot, \cdot)$ is replaced by some approximating quadrature $(\cdot, \cdot)_{h}$ which is of $O\left(h^{q}\right), q \geqq 2 r$, i.e.,

$$
\left|(\alpha, \beta)-(\alpha, \beta)_{h}\right| \leqq C(\alpha, \beta) h^{q}, \quad q \geqq 2 r .
$$

Examples are the extended $r$-point Gauss-Legendre rule or the extended $(r+1)$-point Lobatto rule.

3. Collocation methods. We consider the $m$ th order boundary problem

$$
\begin{aligned}
& L u(x) \equiv D^{m} u(x)+\sum_{i=0}^{m-1} p_{i}(x) D^{i} u(x)=f(x), \quad x \in I, \\
& \beta_{l}[u]=0, \quad l=1, \cdots, m,
\end{aligned}
$$

where $p_{0}, \cdots, p_{m-1}$ and $f$ are sufficiently smooth functions and where $\beta_{1}, \cdots, \beta_{m}$ are continuous linear functionals over $C^{m-1}(I)$. We note that the functions $p_{0}, \cdots, p_{m-1}$ and $f$ and the operator $L$ are not the same as in the previous section. We assume that (3.1) has a unique solution and that $\beta_{1}, \cdots, \beta_{m}$ are linearly independent over $P_{m-1}(I)=\operatorname{ker}\left(D^{m}\right)$.

Let $\Delta$ be a partition of $I$ defined by (1.2). Then, for $k \geqq 2 m-1$, we define the finite element space $S_{0}^{k, m}(\Delta)$ by

$$
\begin{aligned}
& S_{0}^{k, m}(\Delta)=\left\{V \mid V \in C_{0}^{m-1}(I) ; V \in P_{k}\left(I_{j}\right), j=1, \cdots, N\right\}, \\
& C_{0}^{m-1}(I)=\left\{v \mid v \in C^{m-1}(I) ; \beta_{l}[v]=0, l=1, \cdots, m\right\} .
\end{aligned}
$$

The collocation solution $U \in S_{0}^{k, m}(\Delta)$ of (3.1) is defined as follows. 
For $r=k+1-m$, we define the collocation points $z_{j l}$ by

$$
z_{j l}=x_{j-1}+\frac{h}{2}\left(1+\sigma_{l r}^{0}\right), \quad j=1, \cdots, N, \quad l=1, \cdots, r,
$$

where $\left\{\sigma_{l r}^{0}\right\}$ are the zeros of the $r$ th degree Legendre polynomial $P_{r}^{0}(\sigma)$. Then $U$ is determined by the linear system

$$
L U\left(z_{j l}\right)=f\left(z_{j l}\right), \quad j=1, \cdots, N, \quad l=1, \cdots, r .
$$

If $u \in C_{0}^{m-1}(I) \cap C^{2 r+m}(I)$, the error function $e=u-U$ has the bounds [5]

$$
\begin{aligned}
& \|e\|_{W^{l}(I)} \leqq C_{1}(u) h^{k+1-l}, \quad l=0, \cdots, m, \\
& \left|D^{l} e\left(x_{j}\right)\right| \leqq C_{2}(u) h^{2 r}, \quad l=0, \cdots, m-1, \quad j=0, \cdots, N .
\end{aligned}
$$

In order to establish superconvergence at interior points of $I_{j}$ [8], we recall the $n$-dimensional subspace $S_{0}\left(I_{j}\right)$ of $S_{0}^{k, m}(\Delta)$ defined by $(2.15)$. For any $V \in S_{0}\left(I_{j}\right)$, we have, if we put $p_{m}(x) \equiv 1$,

$$
\left(e, L^{T} L V\right)=(L e, L V)+\sum_{l=1}^{m} \sum_{\nu=0}^{l-1}(-1)^{\nu+l}\left[D^{l-\nu-1} e D^{\nu}\left(p_{l} L V\right)\right]_{x_{j-1}^{x_{i}}}^{x_{i}},
$$

where the operator $L^{T}$ is defined by

$$
L^{T} v=\sum_{l=0}^{m}(-1)^{l} D^{l}\left(p_{l} v\right) .
$$

If we apply Lemma 1 to the left-hand side of (3.6), we have

$$
\begin{gathered}
\frac{h}{2} \sum_{l=1}^{n} \omega_{l} e\left(\xi_{j l}\right) L^{T} L V\left(\xi_{j l}\right)+\sum_{l=0}^{m-1}\left[\theta_{l 1} D^{l}\left(e L^{T} L V\right)\left(x_{j-1}\right)+\theta_{l 2} D^{l}\left(e L^{T} L V\right)\left(x_{j}\right)\right]\left(\frac{h}{2}\right)^{l+1} \\
=\left(e, L^{T} L V\right)-R_{m n}\left(\frac{h}{2}\right)^{2 r+1} h^{2 r+1} D^{2 r}\left(e L^{T} L V\right)\left(\xi \in I_{j}\right),
\end{gathered}
$$

where $R_{m n}$ is defined by (2.9).

If we apply the $r$-point Gauss-Legendre rule to the first term of the right-hand side of (3.6), we obtain ([1, formula 25.4.29])

$$
\frac{h}{2} \sum_{l=1}^{r} \lambda_{l r}^{0} L e\left(z_{j l}\right) L V\left(z_{j l}\right)=(L e, L V)-S_{r} h^{2 r+1} D^{2 r}(L e L V)\left(\xi \in I_{j}\right),
$$

$$
S_{r}=\frac{(r !)^{4}}{(2 r+1)[(2 r) !]^{3}} .
$$

In virtue of (3.4), the left-hand side of (3.9) is identically zero. If we combine (3.7)-(3.9) and apply it for the Lagrange basis functions $\phi_{i}$ of $S_{0}\left(I_{j}\right)$ as defined by (2.16), we get after multiplication by $2 h^{2 m-1}$

$$
\begin{aligned}
&\left|\sum_{l=1}^{n} \omega_{l} L^{T} L \phi_{i}\left(\xi_{j l}\right) h^{2 m} e\left(\xi_{j l}\right)\right| \\
& \leqq C_{1} \sum_{l=0}^{m-1}\left(\left|D^{l} e\left(x_{j-1}\right)\right|+\left|D^{l} e\left(x_{j}\right)\right|\right) \\
&+h^{2 m}\left|\sum_{l=0}^{m-1}\left[\theta_{l 1} D^{l}\left(e L^{T} L \phi_{i}\right)\left(x_{j-1}\right)+\theta_{l 2} D^{l}\left(e L^{T} L \phi_{i}\right)\left(x_{j}\right)\right]\left(\frac{h}{2}\right)^{l}\right| \\
&+C_{2} h^{2 k+2}\left[\left\|e L^{T} L \phi_{i}\right\|_{W^{2 r}\left(I_{j}\right)}+\left\|L e L \phi_{i}\right\|_{W^{2 r}\left(I_{j}\right)}\right] \leqq C(u) h^{k+2}, \quad i=1, \cdots, n
\end{aligned}
$$


Analogously to (2.28), we can prove that

$$
\left|\omega_{l} L^{T} L \phi_{i}\left(\xi_{j l}\right) h^{2 m}-2 h^{2 m-1}\left(L \phi_{i}, L \phi_{l}\right)\right| \leqq C h^{2},
$$

which means that, for sufficiently small $h$, the matrix $\left(\omega_{l} L^{T} L \phi_{i}\left(\xi_{j l}\right)\right)$ is an $O\left(h^{2}\right)$ perturbation of the positive definite matrix $\left(2 h^{2 m-1}\left(L \phi_{i}, L \phi_{l}\right)\right.$, whose eigenvalues and entries are of $O(1)$. This implies that the entries of $\left(\omega_{l} L^{T} L \phi_{i}\left(\xi_{j l}\right) h^{2 m}\right)^{-1}$ are of $O(1)$.

THEOREM 3. Let $u \in C_{0}^{m-1}(I) \cap C^{2 r+m}(I)$ be the solution of (3.1) and let $U \in$ $S_{0}^{k, m}(\Delta)$ be the solution of (3.4). Then $e(x)=u(x)-U(x)$ has the bounds (3.5) plus the bounds

$$
\begin{aligned}
& \left|e\left(\xi_{j l}\right)\right| \leqq C(u) h^{k+2}, \quad j=1, \cdots, N, \quad l=1, \cdots, n, \\
& \left|D e\left(\eta_{j l}\right)\right| \leqq C(u) h^{k+1}, \quad j=1, \cdots, N, \quad l=1, \cdots, n+1,
\end{aligned}
$$

where $\xi_{j l}$ and $\eta_{j l}$ are given by (2.18) and (2.35), respectively.

Proof. The first part of (3.12) was already established by (3.9)-(3.11). The second part is proved analogously to Theorem 2 .

Remark. Russell and Christiansen [8] also gave a proof of (3.12); they proved in another way that the first bound of (3.12) occurs at the interior zeros of the polynomial

$$
\int_{-1}^{\sigma}(t-\sigma)^{m-1} P_{r}^{0,0}(t) d t, \quad \sigma=\frac{2}{h}\left(x-x_{j-1}\right)-1,
$$

which can be shown to be equal to $\left(1-\sigma^{2}\right)^{m} P_{n}^{m, m}(\sigma)$ up to a constant factor. The proof of this equality can be given by using formula (2.6) with $\alpha=\beta=0$ and elaborating the integral (3.13) which gives the desired result.

4. Conclusions. In this paper, it was proved for two classes of Galerkin methods that superconvergence also occurs outside the knots of the partition, albeit in a more modest form. Its existence can easily be proved for other classes of problems which are solved by the Ritz-Galerkin or the collocation method. Examples are nonlinear two-point boundary problems and parabolic equations in one space variable [4].

The interior superconvergence is especially important if the finite element space is of degree $2 m$, because the order of convergence at $\bar{x}_{j}$ is then the same as at $x_{j}$.

Appendix. For the computation of $R_{m n}$ from (2.9), we apply that relation to $f \in P_{2 r}(I)$ defined by

$$
f(\sigma)=\left(1-\sigma^{2}\right)^{m}\left[P_{n}^{m}(\sigma)\right]^{2},
$$

where we assume that $P_{n}^{m}(\sigma)$ is normalized in such a way that (see [1, formula 22.2.1])

$$
P_{n}^{m}(1)=\left(\begin{array}{c}
n+m \\
n
\end{array}\right)
$$

From [1, formula 22.2.1], we learn that

$$
\int_{-1}^{+1} f(\sigma) d \sigma=h_{m n}=\frac{2^{2 m+1}(r !)^{2}}{(2 r+1) n !(n+2 m) !} .
$$

From [1, formulas 22.5.42 and 15.1.1], we learn that

$$
P_{n}^{m}(\sigma)=\left(\begin{array}{c}
n+m \\
n
\end{array}\right) \sum_{k=0}^{n} \frac{(-n)_{k}(n+2 m+1)_{k}}{(1+m)_{k} k !}\left(\frac{1-\sigma}{2}\right)^{k},
$$

$$
\begin{aligned}
(a)_{0} & =1, \\
(a)_{k} & =\frac{a(a+1) \cdots(a+k-1)}{k !}, \quad k>0,
\end{aligned}
$$


which means that the coefficient of $\sigma^{2 r}$ in the expression of $f$ is equal to

$$
\begin{aligned}
b_{2 r} & =(-1)^{m} A_{n}^{2}, \\
A_{n} & =\left(\begin{array}{c}
n+m \\
n
\end{array}\right) \frac{(-n)_{n}(n+2 m+1)_{n}}{(1+m)_{n} n !}(-1)^{n} 2^{-n} \\
& =\frac{(n+m) !}{n ! m !} \cdot \frac{(n+2 m+1)(n+2 m+2) \cdots(2 n+2 m)}{(m+1) \cdots(m+2) \cdots(m+n)} 2^{-n} \\
& =2^{-n}\left(\begin{array}{c}
2 n+2 m \\
n
\end{array}\right) .
\end{aligned}
$$

Application of (2.9) to $f$ shows that

$$
h_{m n}=\int_{-1}^{+1} f(\sigma) d \sigma=\int_{-1}^{+1} \Pi f d \sigma+R_{m n} D^{2 r} f(\xi)=0+R_{m n}(2 r) ! b_{2 r}
$$

which implies that

$$
R_{m n}=\frac{h_{m n}}{(2 r) ! b_{2 r}}
$$

Application of (A3) and (A5) to (A7) delivers the desired expression for $R_{m n}$.

\section{REFERENCES}

[1] M. ABRAMOwitz AND I. A. STEGUn, Handbook of Mathematical Functions, National Bureau of Standards Applied Mathematics 55, US Government Printing Office, Washington DC, 1964

[2] M. BAKKER, The Galerkin method for the solution of mildly nonlinear two-point boundary value problems, MC Report NW 27/76, Mathematisch Centrum, Amsterdam, 1976.

[3] — A note on $C^{0}$ Galerkin methods for two-point boundary problems, Numer. Math., 38 (1982), pp. $447-453$.

[4] - Galerkin methods for even-order parabolic problems in one space variable, this Journal, 19 (1982), pp. 571-587.

[5] C. DE Boor AND B. SwarTZ, Collocation at Gaussian points, this Journal, 10 (1973), pp. 582-606.

[6] P. G. CIARLET AND P. A. RAviART, General Lagrange and Hermite interpolation in $R^{N}$ with applications to finite element methods, Arch. Rat. Mech. Anal., 46 (1972), pp. 177-199.

[7] J. Douglas, JR. AND T. DUPONT, Galerkin approximation for the two-point boundary problem using continuous piecewise polynomial spaces, Numer. Math., 22 (1974), pp. 99-109.

[8] R. D. RUSSELL AND J. CHRISTIANSEN, Error analysis for spline collocation methods with application to knot selection, Math. Comp., 32 (1978), pp. 415-419.

[9] M. F. WhEeler, G. F. CAREY AND D. HuMPHREY, Galerkin and collocation-Galerkin method with superconvergence and optimal fluxes, Internat. J. Numer. Meth. Engrg., 17 (1981), pp. 939-950. 